\title{
A study on the dependence of structure of multi-walled carbon nanotubes on acid treatment
}

\author{
S. Sahebian ${ }^{1} \cdot$ S. M. Zebarjad ${ }^{2} \cdot$ J. vahdati Khaki ${ }^{1} \cdot$ A. Lazzeri $^{3}$
}

Received: 26 March 2015 / Accepted: 2 June 2015/Published online: 29 June 2015

(c) The Author(s) 2015. This article is published with open access at Springerlink.com

\begin{abstract}
In the current research, the role of both concentrated nitric acid and ultrasound waves on oxidation of multi-walled carbon nanotubes (MWNTs) was studied. The functionalized MWCNTs were characterized by transmission electron microscopy (TEM), thermogravimetric analyzer, and Fourier transform infrared spectroscopy (FTIR) techniques. It was found that desirable modifications to MWNTs occurred after acid treatment. Carboxylic acid groups were appeared on the side surfaces of MWNTs. FTIR presented the formation of oxygen-containing groups such as $\mathrm{C}=\mathrm{O}$ and $\mathrm{COOH}$ after modification by concentrated nitric acid. The TEM images showed that the aspect ratio of opened MWCNTs was controlled by both ultrasonic waves and acid treatment time. It was also found that the exposure of about $4 \mathrm{~h}$ in nitric acid led to the highest removal of the impurities with the least destructive effect.
\end{abstract}

Keywords Multi-walled carbon nanotube $\cdot$ Structure Acid treatment . Ultrasonic waves

S. M. Zebarjad

mojtabazebarjad@shirazu.ac.ir

1 Department of Materials Science and Engineering, Engineering Faculty, Ferdowsi University of Mashhad, Azadi Square, Mashhad, Iran

2 Department of Materials Science and Engineering, Engineering Faculty, Shiraz University, Namazi Square, Shiraz, Iran

3 Department of Chemical Engineering, University of Pisa, Via Diotisalvi, 56126 Pisa, Italy

\section{Introduction}

Multi-walled carbon nanotubes (MWNTs) have extreme physical, mechanical, and electrical properties which have attracted many potential applications in industry and intensive researches. But low purity of the MWNTs and hydrophobic and inert nature of the surface of MWNTs reduce their applications. The oxidation of MWNTs by using wet chemical [1-9], photo oxidation [10-13], gas phase treatment [13], and oxygen plasma [10] method is a basic technique for purification and also improvement of the chemical reactivity of the MWNTs.

Through the above treatment, the pure MWNTs can be purified and oxygen-containing group, mainly carboxyl, and hydroxyl groups created on the surface of MWNTs. The exfoliation of MWNTs bundles, as functional group, increases the solubility of MWNTs in polar media [2, 3], and more easily incorporated in a polymer matrix. The treatment of MWNTs is more widely done by aqueous solution of acids or oxidizing agents such as nitric acid [14, 15], sulfuric acid [16], $\mathrm{H}_{2} \mathrm{SO}_{4} / \mathrm{HNO}_{3}[17,18], \mathrm{H}_{2} \mathrm{O}_{2}$ [17], etc.

Acid treatment by using $\mathrm{HNO}_{3}$ has been most often used, and it has been divided into two categories: (1) treatment with boiling diluted $\mathrm{HNO}_{3}$, and (2) treatment with pure $\mathrm{HNO}_{3}$, and a mixture of concentrated $\mathrm{H}_{2} \mathrm{SO}_{4} /$ $\mathrm{HNO}_{3}$ in an ultrasonic bath [19]. At both methods, the nitric acid opens the end caps of MWNTs, creates the carboxylic groups at the open ends and the side wall of MWNTs, also introduces the damage on the walls of MWNTs, and even can cut the MWNTs into short of pieces $[5,20]$. So the oxidation condition of MWNTs should be chosen carefully to avoid losing the valuable materials, and introducing further functional groups on the surface of MWNTs. 
Chen et al. [2] showed the opening of end caps, formation of holes in the side walls of MWNTs, and shortening of MWNTs by using ultrasonic treatment in a mixture of $\mathrm{H}_{2} \mathrm{SO}_{4} / \mathrm{HNO}_{3}$ [2]. Goyanes et al. [21] reported that there was no any significant effect on the side walls of MWNTs whenever the exposure time in $\mathrm{HNO}_{3}$ is so short. When the reaction time increased, the carboxyl, hydroxyl, and $\mathrm{C}-\mathrm{O}$ groups appear in the open ends of MWNTs and at extra reaction time, it would begin to destroy the side walls and shorten the length of MWNTs [21].

The main variable of researches in subject of acid treatment of CNTs was classified to composition and concentration of acids, and refluxed time. Ultrasonic waves are commonly used to improve the solubility of CNTs in media as high energy mixer. If it was selected as mixing factor, time that CNTs were dispersed in media must be precisely defined, and implying that mixing by using ultrasonic waves at short time may create defects on the structure of CNTs. In order to find out the role of ultrasonic waves time in acid treatment on structure of MWNTs, mixture of MWNTs and nitric acid was sonicated for different time. Transition electron microscopy (TEM), thermal gravimetric analysis (TGA), X-ray diffraction (XRD), Raman, and Fourier transform infrared spectroscopy (FTIR) techniques were used to evaluate the structure, morphology, and quality of pure and functionalized MWNTs.

\section{Experimental}

\section{Materials}

Multi-walled carbon nanotube (MWNT) with specific diameter of 40-60 nm, specific length of 5-15 $\mu \mathrm{m}$, and purity greater than $95 \%$ was obtained from Nano lin, china.

\section{Acid treatment of MWNTs}

$0.1 \mathrm{~g}$ MWNTs was dispersed in $50 \mathrm{ml}$ concentrated $\mathrm{HNO}_{3}$ (68 wt $\%)$, the mixture was sonicated in a water bath for different time i.e., 1, 2, and $4 \mathrm{~h}$ at ambient temperature, and then stirred at same temperature for $2 \mathrm{~h}$. Afterwards, the mixture was filtered and washed by deionized water for several times until no acid on the sample was detected or up to $\mathrm{pH}$ 6. Opened MWNTs were dried at $90{ }^{\circ} \mathrm{C}$ for overnight. The functionalized MWNTs at different sonication time periods were named as F-MWNTs- $1 \mathrm{~h}$, F-MWNTs-2 h, and F-MWNTs-4 h.

\section{Instruments}

TEM observation with line resolution of $3 \AA$ and operation voltage at $120 \mathrm{kV}$ was carried out using a TEM LEO 912 AB. FTIR was performed by a magna-IR 560 E.S.P FTIR spectrometer. The thermo gravimetric analysis (TGA) was studied using a thermal analysis system (TGA50, Shimadzu) in air atmosphere. Raman spectroscopy measurement was detected by using Almega Thermo Nicolet instrument in domain wave number of $100-4200 \mathrm{~cm}^{-1}$. Solution stability of pure and functionalized carbon nanotube was measured by a Cecil $8000 \mathrm{UV}-\mathrm{V}$ is spectroscopy analysis.

\section{Results and discussion}

Figure 1 shows the TEM micrographs of pure MWNTs (PMWNTs) at different magnification powers. It can be seen that the inner diameter of P-MWNTs was in the range of 5-20 nm and outer diameter was in the range of 40-60 nm. Besides, almost all the end caps of P-MWNTs were closed.
Fig. 1 TEM micrographs of used MWNTs at different magnification powers
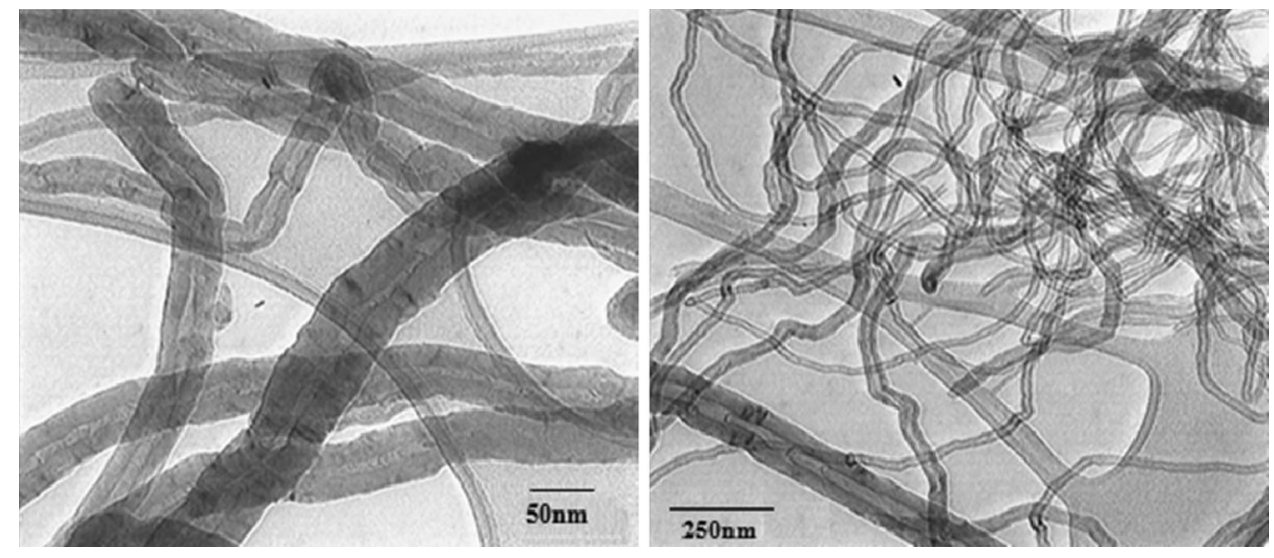
Fig. 2 TEM images of a FMWNT-2 h, and b F-MWNT$4 \mathrm{~h}$
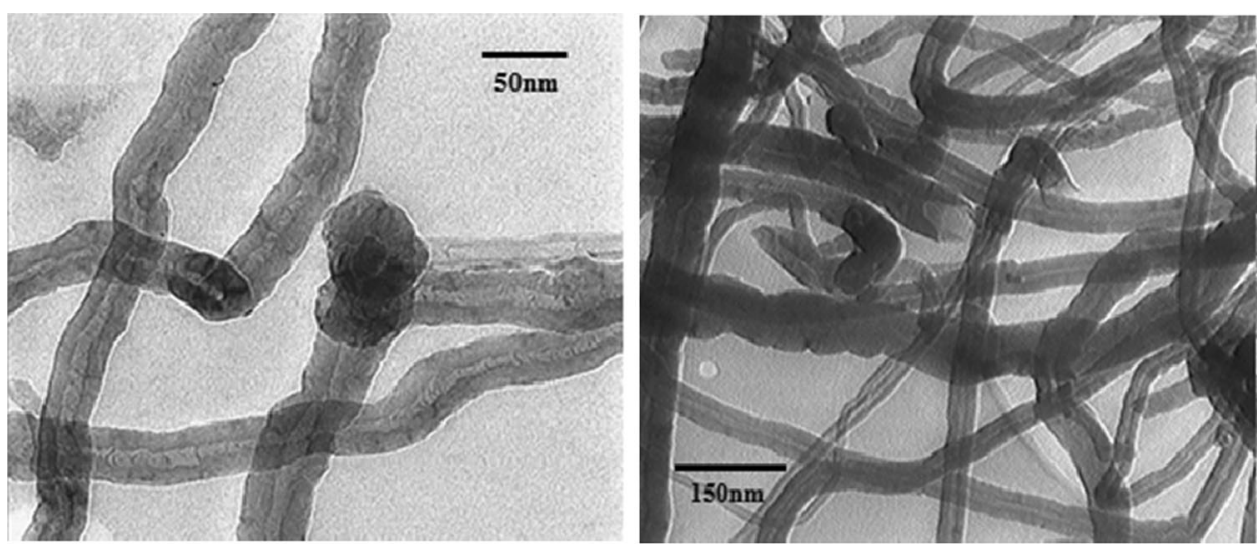

(a)
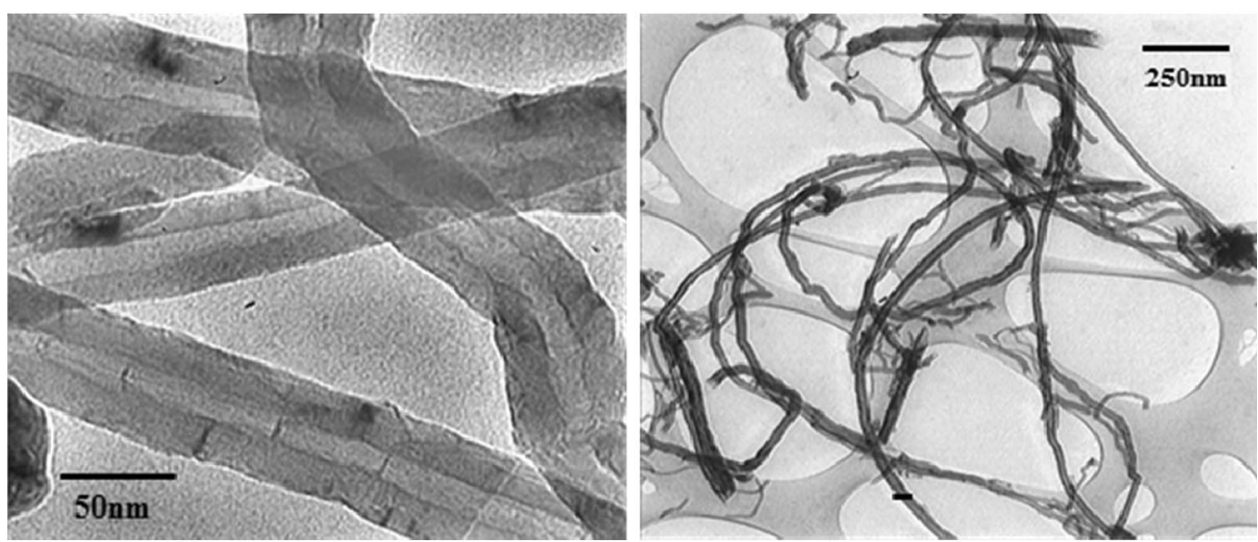

(b)

The effect of acid treatment condition (exposure time) on the morphology of MWNTs is presented in Fig. 2a, b. As shown in these figures, almost all the end caps of F-MWNT-2 $\mathrm{h}$ sample are opened and do not see the harmless effect on the surface of MWNTs. Whenever the soaking time of acid treatment increases, the defect sites in the outside appear, besides it can be seen some short length MWNTs in F-MWNT-4 h sample compared to others.

In order to find the best condition for functionalization of carbon nanotube with minimum defect on their structures, TGA was used. Figure 3 shows the weight loss and derivative weight loss of P-MWNT and functionalized MWNTs. As seen the degradation of P-MWNT occurs in one step at a temperature range of $610-710{ }^{\circ} \mathrm{C}$. The weight loss below $150{ }^{\circ} \mathrm{C}$ is related to water loss, between 150 and $400{ }^{\circ} \mathrm{C}$ is due to carboxyl groups degradation. Finally weight loss at higher temperature $400{ }^{\circ} \mathrm{C}$ is due to carboxyl groups degradation. Finally weight loss at higher temperature $400{ }^{\circ} \mathrm{C}$ it is referred to the degradation of oxygen atoms tightly bonded to the surface of the MWNTs or nongraphitic carboneous species [22]. As seen in the Figure there is no any significant weight loss before $500{ }^{\circ} \mathrm{C}$ in the P-MWNTs and implies that a significant amount of amorphous carbon and carboxyl group rules out.

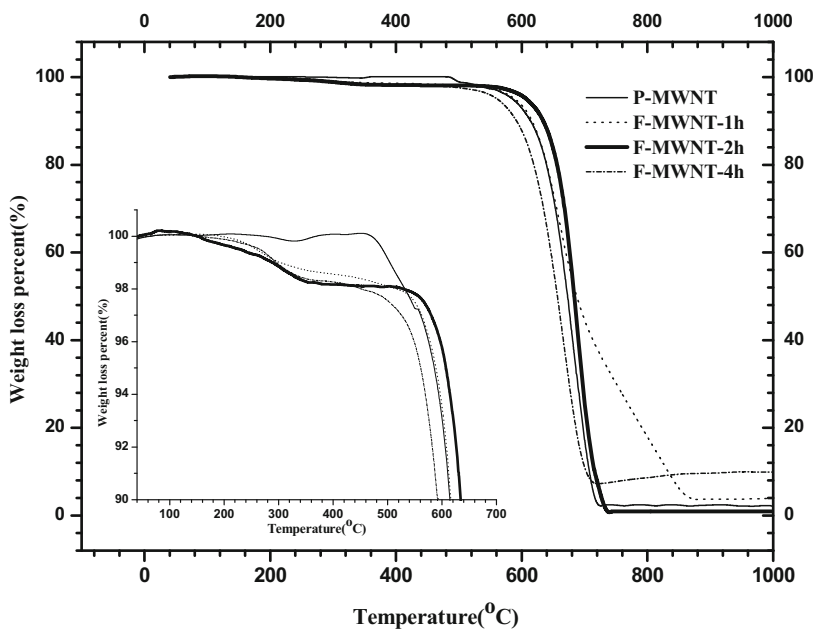

Fig. 3 Weight loss of P-MWNT, F-MWNT-1 h, F-MWNT-2 h, and F-MWNT-4 $\mathrm{h}$ as a function of temperature

As seen Fig. 3, increasing temperature from room temperature up to $800{ }^{\circ} \mathrm{C}$ causes to degrade the functionalized MWNTs in two steps. The weight loss until $400{ }^{\circ} \mathrm{C}$ is due to formation of hydroxyl and carboxyl groups on the surface of CNT. Similar to what proposed for P-MWNT 
weight loss at temperature above $400{ }^{\circ} \mathrm{C}$ is referred to the degradation of oxygen atoms tightly bonded to the surface of the MWNTs. Besides, F-MWNT-2 h (of about $2.8 \mathrm{wt} \%$ ) has more weight loss until $400{ }^{\circ} \mathrm{C}$ than F-MWNT-4 h (of about $1.68 \mathrm{wt} \%$ ) sample; it means that the amount of hydroxyl and carboxyl groups on the surface of F-MWNT$2 \mathrm{~h}$ is higher than F-MWNT-4 h, but degradation of F-MWNT-4 h sample starts at lower temperature than others as severe damage was seen in the structure of MWNTs like as reduction of size or diameter and defects of side walls.

Figure 4 depicts the DTG graph of pure and functionalized samples. As seen, by increasing temperature from room temperature up to $800{ }^{\circ} \mathrm{C}$ degradation of MWNTs occurs at two steps. By comparison of second peak of all samples, it can be implied that F-MWNT-2 $\mathrm{h}$ has best thermal stability as maximum degradation temperature and also almost all CNTs in this sample have similar structure and functionalized groups on surface as narrow degradation peak.

The summary of achieved results of TGA and DTG curves including the temperature at initial weight loss ( $\left.T_{\text {onset }}\right)$, maximum weight loss rate $\left(T_{\text {midpoint }}\right)$, and the final weight loss $\left(T_{\text {endset }}\right)$ and residue at $900{ }^{\circ} \mathrm{C}$ are summarized in Table 1. The results show that the position of $T_{\text {onset }}$, $T_{\text {midpoint }}$ appears at higher temperature when the P-MWNT

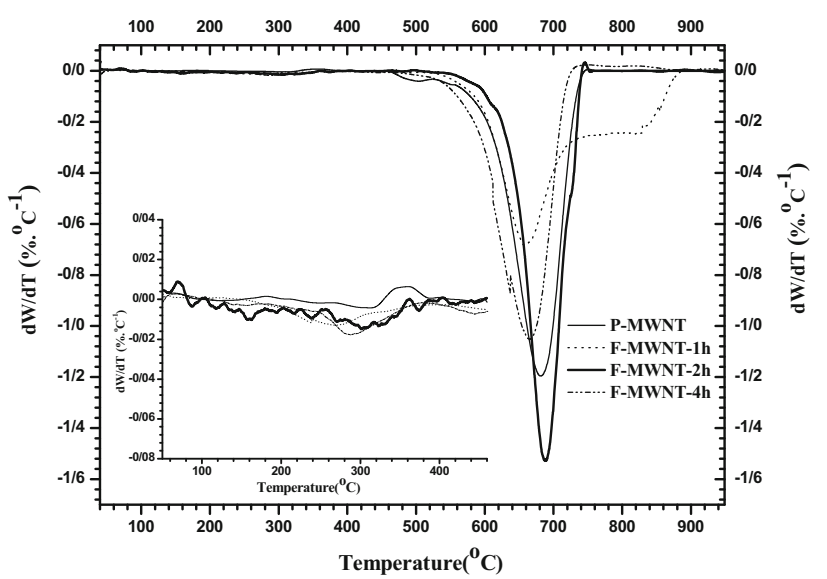

Fig. 4 DTG curve of P-MWNT, F-MWNT-1 h, F-MWNT-2 h, and F-MWNT-4 $\mathrm{h}$ as a function of temperature was acid treated for 1 and $2 \mathrm{~h}$. Perhaps the main reason of this variation can be attributed to the fact that during early oxidation of F-MWNT, the oxidant starts to attack the active sites already existed on the MWNTs such as $\mathrm{CH}_{2}$ and $\mathrm{CH}$ groups, so the thermal stability of F-MWNTs improves [1]. Besides lack of formation defects on surface of CNTs due to acid treatment is another reason for promotion of thermal stability of MWCNTs after acid treatment. Looking at in more details (appeared in Table 1) on the weight loss between 150 and $400{ }^{\circ} \mathrm{C}$ of MWCNTs including pure and functionalized MWNTs, one may conclude that for the materials used in the current research, the best condition of acid treatment is around $2 \mathrm{~h}$ ultrasound and $2 \mathrm{~h}$ stirring at room temperature.

The FTIR spectra of P-MWNT F-MWNT-1 h, F-MWNT-2 h, and F-MWNT-4 h are shown in Fig. 5. P-MWNT shows very low intensity at around 3440, $1711 \mathrm{~cm}^{-1}$ corresponding to $\mathrm{OH}$ and $\mathrm{C}=\mathrm{O}$. $\mathrm{OH}$ peak is related to observed water molecules on the carbon in the CNTs. In the case of F-MWNT, there is an additional peak, appeared at $1719 \mathrm{~cm}^{-1}$ which is due to the $\mathrm{C}=\mathrm{O}$ stretching frequency, indicating the presence of carboxylic group created during the oxidation of MWNTs [1]. The intensity implies more number of carboxylic groups which had been successfully attached to the surface of MWNT after acid treatment. Also there are two peaks around 2923 and $2853 \mathrm{~cm}^{-1}[1,22]$, belonging to $\mathrm{CH}_{2}$ group on the surface of F-MWNT-2 h, and implies the higher stability of F-MWNT-2 $h$ than P-MWNTs. When the time of acid treatment increases, the intensity of hydroxyl and carboxyl groups in spectrum goes toward a high value. The interesting point can be the elimination of $\mathrm{CH}_{2}$ group on the surface of MWNTs. The stability of this sample is lower than that of acid treated samples and pure MWNT.

Figure 6 demonstrates the Raman spectra of P-MWNT, F-MWNT-2 h, and F-MWNT-4 h samples. In Raman spectra of MWNTs, there are three prominent peaks at around $1250-1440 \mathrm{~cm}^{-1}\left(D_{\text {band }}\right)$, and $1500-1600 \mathrm{~cm}^{-1}$ $\left(G_{\text {band }}\right)$ and $282 \mathrm{~cm}^{-1}\left(\mathrm{RBM}^{\prime}\right)$. The first is related to disorder structure bond attributed to the defect in the curved graphene sheet and tube ends or even amorphous carbons. The second peak is referred to the structure integrity of $\mathrm{sp}^{2}$ bonded carbon atoms in two-dimensional hexagonal

Table 1 The details of thermal stability of MWNTs functionalized at different conditions

\begin{tabular}{lllllllcc}
\hline Samples & $T_{\text {onset }}$ & $T_{\text {midpoint }}$ & $T_{\text {endset }}$ & $\begin{array}{l}\text { Weight loss }(\%) \\
\text { between room } \\
\text { temperature and } 400{ }^{\circ} \mathrm{C}\end{array}$ & $T_{\text {onset }}$ & $\begin{array}{c}T_{\text {midpoint }} \\
T_{\text {endset }}\end{array}$ & $\begin{array}{l}\text { Residue }(\%) \\
\text { at } 900{ }^{\circ} \mathrm{C}\end{array}$ \\
\hline P-MWNT & - & - & - & - & 615.69 & 652.37 & 692.34 & 1.7 \\
F-MWNT-1 h & 268.2 & 300.3 & 335.6 & 1.88 & 640.2 & 692.9 & 747.6 & 3.8 \\
F-MWNT-2 h & 272.2 & 302.6 & 340.3 & 2.81 & 630.2 & 684.6 & 739.9 & 0.98 \\
F-MWNT-4 h & 289.1 & 319.2 & 365.5 & 1.68 & 605.9 & 650.0 & 695 & 7.2 \\
\hline
\end{tabular}




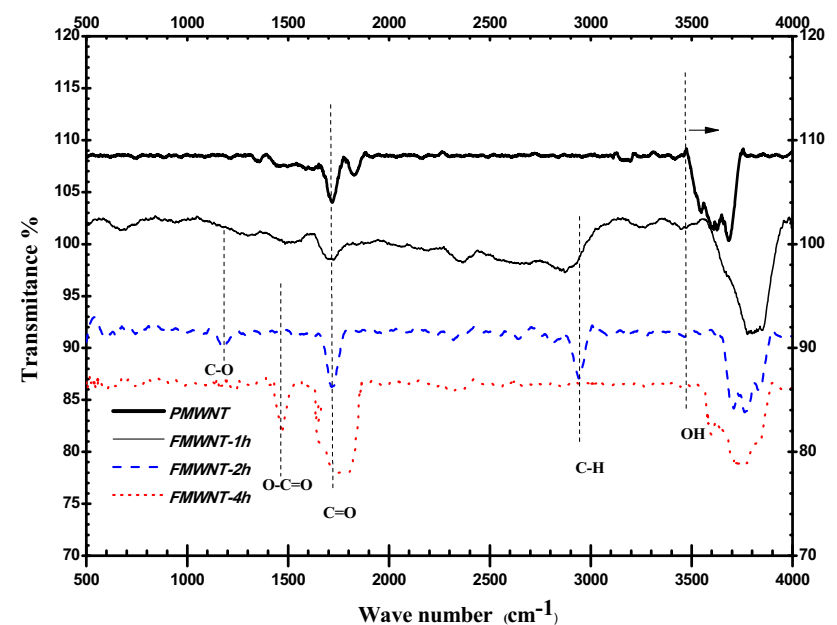

Fig. 5 FTIR spectra of P-MWNT, F-MWNT-1 h, F-MWNT-2 h, and F-MWNT-4 h

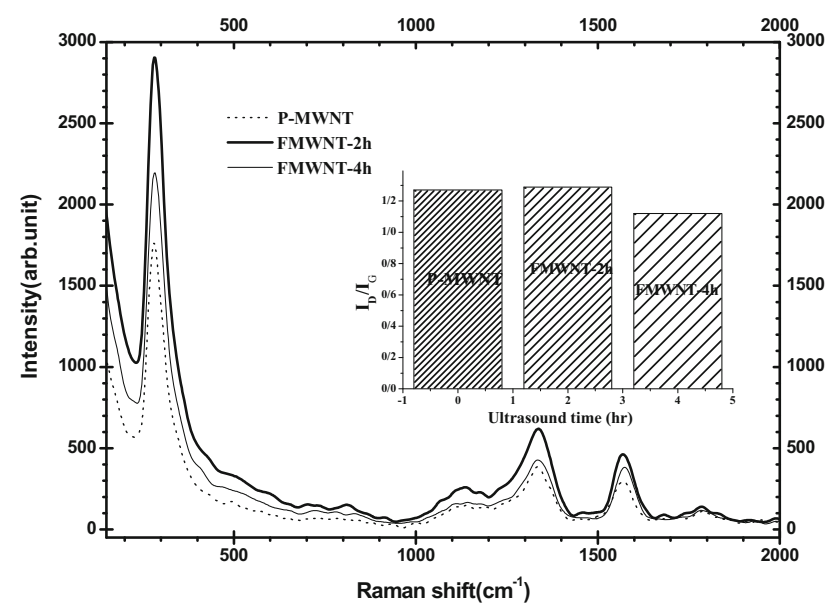

Fig. 6 The Raman spectra of P-MWNT, F-MWNT-2 h, and F-MWNT-4 h samples

lattices, indicating the presence of crystalline graphitic carbon [23]. The $\mathrm{I}_{\mathrm{D}} / \mathrm{I}_{\mathrm{G}}$ indicates the quality of MWNTs, the higher intensity of $I_{\mathrm{D}} / I_{\mathrm{G}}$ ratio is reflective of lower quality of MWNTs. This ratio for P-MWNT, F-MWNT-2 h, and F-MWNT-4 $\mathrm{h}$ are 1.27, 1.29, and 1.12, respectively. The similarity of the ratio for P-MWCNT and F-MWNT-2 h implies that the acid treatment has no big significant effect on the surface of CNTs. Soaking of CNTs for $2 \mathrm{~h}$ in nitric acid causes to open end cap and create some defects on the surface of CNTs, but it leads to remove amorphous carbon and impurity in sample. These two effects have the same weight on the structure of CNTs, and thus can be seen the ratio for these are almost similar. The results of current research indicate that increasing soaking time up to $4 \mathrm{~h}$ causes to decrease the ratio. Wepasnik et al. [24] showed similar results for CNTs that have severe destruction and they claimed that Raman analysis cannot give acceptable

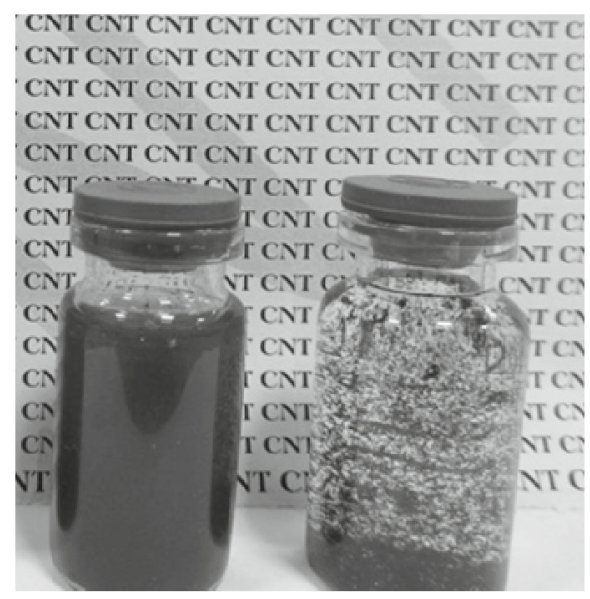

(a) 1 hour

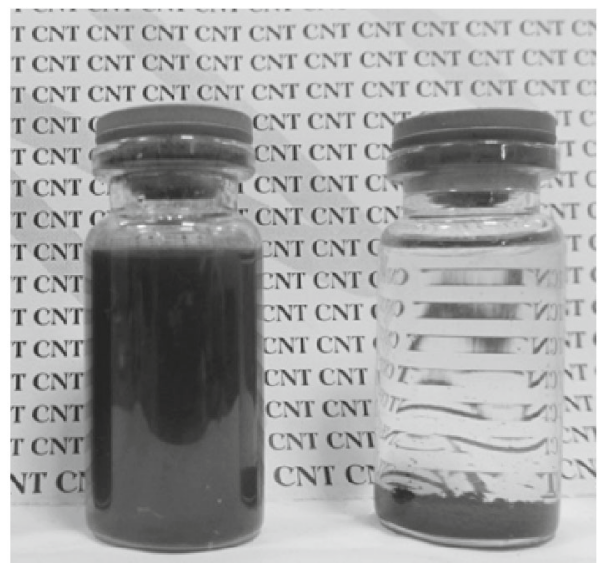

(b) 1 week

Fig. 7 The dependency of dispersibility of MWNTs in water at different time, left F-MWNT-2 $\mathrm{h}$, and right P-MWNTs a $1 \mathrm{~h}$ b 1 week

data for CNTs, which is severely oxidized. Therefore, Raman data of FCNT-4 h cannot be valid.

RBM' band for P-MWNT, F-MWNT-2 h, and F-MWNT-4 h are 281.45, 281.68, and $282.77 \mathrm{~cm}^{-1}$, respectively. According to RBM' band, P-MWNT and FMNT-2 h have same diameter, but FMNT-4 h has lower diameter compared to others, and it can be attributed to severe destruction and removal of last layers of MWNTs during acid treatment.

As the reader knows like other nanoparticles, agglomeration is one of the most limitations for CNTs. In order to avoid agglomeration, the presence of chemical bonds as functional group on the surface of carbon nanotube can be useful. Also these groups such as carboxyl, hydroxyl, and carboxylate, impart negative charges which cause to the electrostatic stability in solution [25]. Figure 7 shows the dependency of dispersibility of MWNTs in water on functionality as a function of time. In order to check the 
Fig. 8 The UV-Vis spectrum of P-MWNTs and F-MWNT$2 \mathrm{~h}$ in water after half an hour and 20 days of 5 min ultrasonic mixing

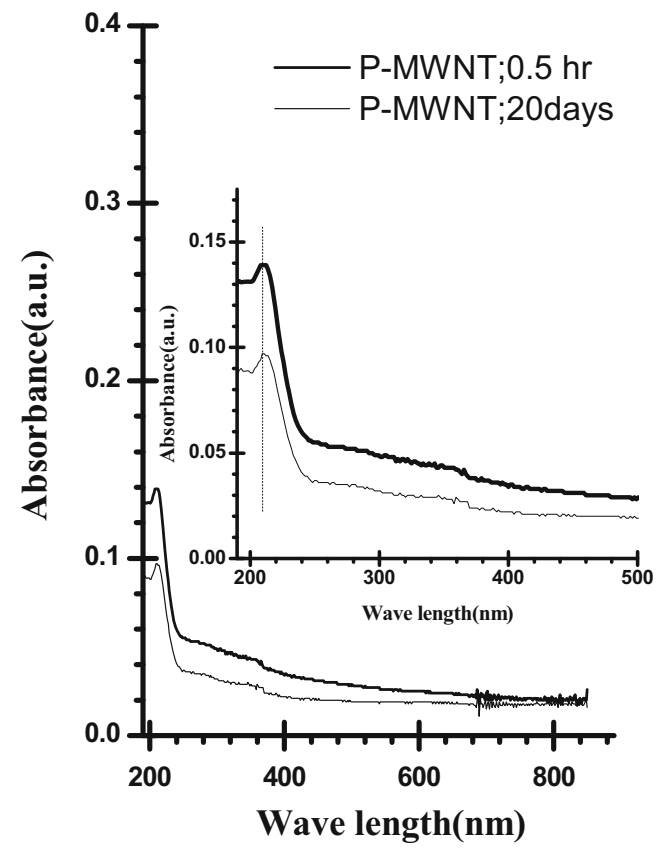

dispersity of these samples at first, MWNTs were dispersed in water by sonication for $5 \mathrm{~min}$, and after that they were kept for $1 \mathrm{~h}$ and 1 week. As seen in the Figure, chemical treatment applied to CNTs improves the dispersion stability of MWNTs in water.

Figure 8 depicts the UV-Vis spectrum of P-MWNTs and F-MWNT-2 $\mathrm{h}$ after half an hour and 20 days exposure. The samples were sonicated 5 min to find out the effect of acid treatment on the dispersibility of CNTs in media like water. The ultra-violet spectrum of P-MWNTs has maximum absorbance around $209.5 \mathrm{~nm}$ and this is related to $\pi-$ $\pi^{*}$ charge transition of $\mathrm{C}-\mathrm{C}$ aromatic bond of CNT structure $[26,27]$. After half an hour of ultrasonic mixing for P-MWNT sample, the absorbance value is low, implying the hydrophobic behavior and low dispersion of CNTs in water. As seen in the figure, after 20 days, the absorbance value is reduced significantly, meaning that the transition optical wave increases and proves the inconstancy of CNTs in water. Also the result presents two peaks around 209.5 and $293.5 \mathrm{~nm}$ as $\mathrm{C}-\mathrm{C}$ aromatic bond and $\mathrm{C}=\mathrm{O}$ bond, respectively, for F-MWNT-2 h sample. Indeed the value absorbance of F-MWNTs is much more than P-MWNTs because of better dispersion in water. This effect is related to the formation of carboxyl and hydroxyl groups on the surface of CNTs and improvement of hydrophobic behavior of CNTs. Also it appears that the specific peak of pure CNTs is wider than F-MWNT-2 h sample; and after 20 days, the peak wide is increased which is the conclusion of the agglomeration of CNTs and low dispersity of CNTs in water. Briefly, the result proves the presence of functional group on the surface of MWCNT and leads to promote the solubility of MWNTs in water as media.

\section{Conclusion}

In the current research, the role of soaking time of MWCNT into concentrated nitric acid on its surface chemistry was investigated. The results showed that by increasing the oxidation time the number of functional groups and defect sites on the surface of MWNTs increased. The TGA analysis showed that at the beginning of the oxidation, thermal stability of MWNCTs promoted and as soaking time increased it decreased due to cutting the MWCNTs. This behavior was validated using TEM, FTIR, Raman spectra, and ultra-violet spectra.

Open Access This article is distributed under the terms of the Creative Commons Attribution 4.0 International License (http://creativecommons.org/licenses/by/4.0/), which permits unrestricted use, distribution, and reproduction in any medium, provided you give appropriate credit to the original author(s) and the source, provide a link to the Creative Commons license, and indicate if changes were made.

\section{References}

1. Chiang, Y.C., Lin, W.H., Chang, Y.C.: The influence of treatment duration on multi-walled carbon nanotubes functionalized by $\mathrm{H}_{2}$ $\mathrm{SO}_{4} / \mathrm{HNO}_{3}$ oxidation. Appl. Surf. Sci. 257, 2401-2410 (2011)

2. Chen, J., Hamon, M.A., Hu, H., Chen, Y., Rao, A., Eklund, P.C., Haddon, R.C.: Solution properties of single-walled carbon nanotubes. Science 282, 95-99 (1998)

3. Liu, J., Rinzler, A.G., Dai, H.J., Hafner, J.H., Bradley, R.K., Boul, P.J., Lu, A., Iverson, T., Shelimov, K., Huffman, C.B., Rodriguez-Macias, F., Shon, Y.S., Lee, T.R., Colbert, D.T., Smalley, R.E.: Fullerene pipes. Science 280, 1253-1256 (1998)

4. Monthioux, M., Smith, B.W., Burteaux, B., Claye, A., Fischer, J.E., Luzzi, D.E.: Sensitivity of single-wall carbon nanotubes to 
chemical processing: an electron microscopy investigation. Carbon 39, 1251-1272 (2001)

5. Hu, H., Zhao, B., Itkis, M.E., Haddon, R.C.: Nitric acid purification of single-walled carbon nanotubes. J. Phys. Chem. B 107, 13838-13842 (2003)

6. Marti, M.T., Callejas, M.A., Benito, A.M., Cochet, M., Seeger, T., Anson, A., Schreiber, J., Gordon, C., Marhic, C., Chauvet, O., Fierro, J.L.G., Maser, W.K.: Sensitivity of single wall carbon nanotubes to oxidative processing: structural modification, intercalation and functionalisation. Carbon 41, 2247-2256 (2003)

7. Zhang, J., Zou, H.Q., Qing, Y., Yang, Q., Li, Zh, Liu, X., Guo, ZDu: Effect of chemical oxidation on the structure of singlewalled carbon nanotubes. J. Phys. Chem. B 107, 3712-3718 (2003)

8. Ziegler, K.J., Gu, Z., Peng, H., Flor, E.L., Hauge, R.H., Smalley, R.E.: Controlled oxidative cutting of single-walled carbon nanotubes. J. Am. Chem. Soc. 127, 1541-1547 (2005)

9. Rosca, I.D., Watari, F., Uo, M., Akasaka, T.: Oxidation of multiwalled carbon nanotubes by nitric acid. Carbon 43, 3124-3131 (2005)

10. Felten, A., Bittencourt, C., Pireaux, J.J.: Gold clusters on oxygen plasma functionalized carbon nanotubes: XPS and TEM studies. Nanotechnology 17(8), 1954-1959 (2006)

11. Savage, T., Bhattacharya, S., Sadanadan, B., Gaillard, J., Tritt, T.M., Sun, Y.P., Wu, Y., Nayak, S., Car, R., Marzari, N., Ajayan, P.M., Rao, A.M.: Photoinduced oxidation of carbon nanotubes. J. Phys. Condens. Matter 15, 5915-5922 (2003)

12. Grujicic, M., Gao, G., Rao, A.M., Tritt, T.M., Nayak, S.: UVlight enhanced oxidation of carbon nanotubes. Appl. Surf. Sci. 214, 289-303 (2003)

13. Tsang, S.C., Harris, P.J.F., Green, M.L.H.: Thinning and opening of carbon nanotubes by oxidation using carbon dioxide. Nature 362, 520-522 (1993)

14. Moreno-Castilla, C., Carrasco-Marin, F., Maldonado-Hodar, F.J., Rivera-Utrilla, J.: Effects of non-oxidant and oxidant acid treatments on the surface properties of an activated carbon with very low ash content. Carbon 36, 145-151 (1998)

15. Haydar, S., Moreno-Castilla, C., Ferro-García, M.A., CarrascoMarín, F., Rivera-Utrilla, J., Perrard, A., Joly, J.P.: Regularities in the temperature-programmed desorption spectra of $\mathrm{CO}_{2}$ and $\mathrm{CO}$ from activated carbons. Carbon 38, 1297-1308 (2000)

16. Cuentas-Gallegos, A.K., Martínez-Rosales, R., Rincón, M.E., Hirata, G.A., Orozco, G.: Design of hybrid materials based on carbon nanotubes and polyoxometalates. Opt. Mater. 29, 126-133 (2006)

17. Xing, Y., Li, L., Chusuei, C.C., Hull, R.V.: Sonochemical oxidation of multiwalled carbon nanotubes. Langmuir 21, 4185-4190 (2005)

18. Osorio, A.G., Silveira, I.C.L., Bueno, V.L., Bergmann, C.P.:

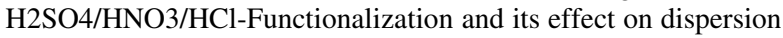
of carbon nanotubes in aqueous media. Appl. Surf. Sci. 255, 2485-2489 (2005)

19. Tchoul, M.N., Ford, W.T., Lolli, G., Resasco, D.E., Arepalli, S.: Effect of mild nitric acid oxidation on dispersability, size, and structure of single-walled carbon nanotubes. J. Mater. Chem. 19, 5765-5772 (2007)

20. Itkis, E.M., Niyogi, S., Meng, M., Hamon, M., Hu, H., Haddon, R.C.: Spectroscopic study of the Fermi level electronic structure of single-walled carbon nanotubes. Nano Lett. 2, 155-159 (2002)

21. Goyanes, S., Rubiolo, G.R., Salazar, A., Jimeno, A., Corcuera, M.A., Mondragon, I.: Carboxylation treatment of multiwalled carbon nanotubes monitored by infrared and ultraviolet spectroscopies and scanning probe microscopy. Diam. Relat. Mater. 16, 412-417 (2007)

22. Figueiredo, J.L., Pereira, M.F.R., Freitas, M.M.A., Orfao, J.J.M.: Modification of the surface chemistry of activated carbons. Carbon 37(9), 1379-1399 (1999)

23. Lehman, J.H., Terrones, M., Mansfield, E., Hurst, K.E., Meunier, V.: Evaluating the characteristics of multiwall carbon nanotubes. Carbon 49(8), 2581-2602 (2011)

24. Wepasnick, K.A., Smith, B.A., Schrote, K.E., Wilson, H.K., Diegelmann, S.R., Fairbrother, D.H.: Surface and structural characterization of multi-walled carbon nanotubes following different oxidative treatments. Carbon 49, 24-36 (2011)

25. Mohammad, F.A., Rapolu, K., Valugonda, S.K., Balusu, S.: Optimization of Cuprous Oxide Nanocrystals Deposition on Multiwalled Carbon Nanotubes. Asian J. Res. Chem. 5, 116-122 (2012)

26. Yu, J., Grossiord, N., Koning, C.E., Loos, J.: Controlling the dispersion of multi-wall carbon nanotubes in aqueous surfactant solution. Carbon 45, 618-623 (2007)

27. Paredes, J.I., Villar-Rodil, S., Martinez-Alonso, A., Tascon, J.M.D.: Graphene oxide dispersions in organic solvents. Langmuir 24, 10560-10564 (2008) 